\title{
Mehr als Dialekt-Relikte: Regionale Variation im Gegenwartsdeutschen
}

\author{
More than Dialect Relics: Regional Variation in Contemporary German
}

\begin{abstract}
ZUSAMMENFASSUNG
Obwohl der Dialektgebrauch in den letzten 100 Jahren in weiten Teilen des Sprachgebiets des Deutschen massiv zurückgegangen ist, besteht insgesamt immer noch eine erhebliche areale Vielfalt. Selbst die geschriebene Standardsprache ist auf verschiedenen Ebenen - Aussprache, Lexik, Grammatik, Pragmatik - von diatopischer Heterogenität geprägt. Dies gilt umso mehr für die gesprochene Alltagssprache, die je nach Land und Gebiet in den deutschsprachigen Ländern mehr dialektal, regiolektal oder standardnah geprägt sein kann. Der vorliegende Beitrag fokussiert auf die lexikalische Variation und präsentiert dazu Daten des Atlas zur deutschen Alltagssprache (AdA) aus Online-Erhebungen der letzten 17 Jahre - zum Teil im Vergleich mit älteren Daten des Wortatlas der deutschen Umgangssprachen (WDU), die in den 1970er Jahren erhoben wurden. Die bisher ca. 600 erstellten Karten des AdA dokumentieren zum einen - bis in den Standard hinein - eine erstaunlich klare Erhaltung älterer regionaler Gegensätze im Wortschatz, wie man sie schon aus früheren Dialektatlanten kennt. Zum anderen zeigen die AdA-Karten eine Vielzahl neuerer Fälle regionaler Diversität, die bisher kaum oder gar nicht bekannt waren und entsprechend in Darstellungen zur Variation in der Lexik des Gegenwartsdeutschen nicht aufgeführt sind. Es wird gezeigt, dass auch Varianten für moderne Begriffe oft nicht regional einheitlich sind, sondern deutliche regionale Schwerpunkte haben können. Abschließend wird der Frage nach dominanten arealen Strukturen in der gegenwärtigen lexikalischen Variation des Deutschen nachgegangen.
\end{abstract}

Schlüsselwörter: Alltagssprache, Lexik, regionale Variation

\section{ABSTRACT}

Although dialect use has declined massively over the past 100 years in large parts of the Germanspeaking countries, there is still a considerable areal diversity overall. Even the written standard language is characterised by diatopic heterogeneity on various levels - pronunciation, lexis, grammar, pragmatics. This is even more true for spoken everyday language, which, depending on the country and area, may be more dialectal, regiolectal, or near-standard in the Germanspeaking countries. This paper focuses on lexical variation and presents data from the Atlas zur deutschen Alltagssprache (AdA) from online surveys conducted over the last 17 years; some of these data is compared with older data from the Wortatlas der deutschen Umgangssprachen

Robert Möller, Département de Langues modernes, Centre d'Études Allemandes, Université de Liège, Place Cockerill, 3, 4000 Liège, Phone: 0032 043665448, r.moller@uliege.be,

https://orcid.org/0000-0002-7996-1714

Stephan Elspaß, Fachbereich Germanistik, Universität Salzburg, Erzabt-Klotz-Str. 1, 5020 Salzburg, stephan.elspass@sbg.ac.at, https://orcid.org/0000-0002-3870-0817 
(WDU) collected in the 1970s. The approx. 600 maps of the AdA produced so far document, on the one hand, a surprisingly clear preservation of older regional contrasts in the distribution of diatopic variants, as already known from earlier dialect atlases. On the other hand, the AdA maps show a multitude of newer cases of regional diversity, which were hardly or not at all known before and which are thus not listed in codices or studies on the lexis of contemporary German. The paper shows that even variants for modern concepts are often not uniform across regions but can have distinct regional emphases. Finally, the question of dominant areal structures in presentday lexical variation of German will be addressed.

Keywords: everyday language, lexis, regional variation

\section{Einleitung}

Mit dem massiven Rückgang des Dialektgebrauchs in einem großen Teil des Sprachgebiets ist die areale Vielfalt des im Alltag verwendeten Deutschen zwar heute nicht mehr so groß wie vor 100 Jahren. Die Vorstellung eines überregional einheitlichen, allenfalls noch nach nationalen Varietäten differenzierbaren Sprachgebrauchs ist jedoch nach wie vor weit von der Realität der deutschen Sprache der Gegenwart entfernt. Dies gilt sogar noch für die geschriebene Standardsprache und die Aussprache nach der Schrift, wie das Variantenwörterbuch (VWB) ${ }^{1}$, die Variantengrammatik (VG) ${ }^{2}$ sowie der Atlas zur Aussprache des deutschen Gebrauchsstandards (AADG) ${ }^{3}$ eindrücklich zeigen, und erst recht für die Alltagssprache i.S. einer informellen gesprochenen Sprache des Nähebereichs, die der Atlas zur deutschen Alltagssprache (AdA) erhebt und dokumentiert.

Der Schwerpunkt des AdA liegt auf der Lexik. In bislang ca. 600 erstellten Karten ist zunächst einmal - trotz deutlicher Tendenz zum Variantenabbau - oft eine erstaunlich klare Erhaltung älterer regionaler Gegensätze zu erkennen, die schon in Dialektatlanten dokumentiert sind. Darüber hinaus zeigt sich jedoch, dass auch Bezeichnungen für moderne Begriffe oft nicht überregional einheitlich sind. Im Folgenden stehen solche Phänomene und die dabei dominierenden Arealstrukturen im Zentrum.

\section{Die gegenwärtige lexikalische Variation im Deutschen}

\subsection{Standardsprachliche Variation}

Bekanntermaßen hat der Abbau regionaler Heteronymik im Standarddeutschen bis heute nicht den Stand durchgehender sprachraumweiter Einheitlichkeit erreicht. Über einige immer wieder angeführte Beispiele wie Brötchen/Semmel/Weck (vgl. AdA 9-1hi') hinaus koexistieren für zahlreiche Begriffe weiterhin verschiedene

\footnotetext{
1 Ammon et al. (2016).

2 Variantengrammatik des Standarddeutschen (2018).

Von Kleiner (2011-2021).

4 Auf die entsprechenden AdA-Karten wird im Folgenden mit Siglen referiert: Die erste Zahl bezieht sich auf die Fragerunde, die zweite - gegebenenfalls mit folgendem Kleinbuchstaben -
} 
landschaftliche Heteronyme. Nach der Online-Version des Duden-Wörterbuchs (im Folgenden kurz: Duden-Wb.) sowie nach dem VWB gibt es z.B. keine nicht regional gebundene Bezeichnung für Rotkohl/Rotkraut/Blaukraut: Rotkohl gilt im Duden-Wb. als „bes. norddeutsch“, und die beiden anderem Varianten werden als „süddeutsch, österreichisch“ spezifiziert (vgl. www.duden.de); im VWB finden sich folgende Markierungen: Rotkohl „CH D“, Rotkraut „A CH D-mittel/ südwest“, Blaukraut „A D-mittelwest/süd“. Daneben gibt es den Fall, dass Varianten im Duden-Wb. ohne diatopische Markierung erscheinen, allerdings eine klare regionale Verteilung aufweisen, z.B. die Wörter Portemonnee und Geldbeutel (vgl. AdA 8-3i). Der Befund ist am Ende derselbe: Einen überregional einheitlichen standardsprachlichen Gebrauch gibt es in vielen Fällen nicht.

Ein Problem stellt diese regionale Variation in der Lexik z.B. für den Handel dar, bei Produktbezeichnungen. Insbesondere der Online-Handel muss dafür sorgen, dass die Eingabe von Suchbegriffen potenzielle Kunden zu den Produkten führt. Eine nicht selten anzutreffende Lösung dafür entspricht einem schon in der Frühzeit der Standardisierung des Deutschen verwendeten Verfahren (vgl. Hartweg \& Wegera, 2005, S. 185): Es wird mit Addition der Varianten gearbeitet. Diese Technik erscheint etwa auch in den Stuttgarter Nachrichten, wenn es heißt: „So vertreiben Sie wirkungsvoll Mücken und Schnaken. “5 Hier geht es wohl nicht um die Unterscheidung zwischen zwei Insektenarten, sondern um die Kombination der nach dem Duden-Wb. überregionalen Bezeichnung Mücke mit der südwestdeutschen Bezeichnung Schnake für dasselbe Insekt (denn das im Mücke-Gebiet Schnake genannte Insekt belästigt Menschen gar nicht ${ }^{6}$ ).

Das Beispiel deutet hier allerdings - neben der Verdrängung von Varianten durch andere - auf einen weiteren Weg zur Vereinheitlichung hin, nämlich auf die (mögliche) Entwicklung in Richtung Bedeutungsdifferenzierung. Dass ehemalige landschaftliche Heteronyme mit unterschiedlicher Bedeutung nebeneinander in der Standardsprache erhalten sind, also der Abbau diatopischer Heterogenität mit dem Ausbau der lexikalischen Differenzierungsmöglichkeiten einhergeht, ist u.a. gerade bei Waren- und Produktbezeichnungen zu beobachten, wie etwa bei Topfen vs. Quark oder Sahne vs. Schmand (vgl. auch Möller \& Elspaß,

auf die einzelnen Karten der jeweiligen Runde; nach dieser zweiten Zahl sind die Karten zu einer Fragerunde alphabetisch geordnet. „AdA 9-1hi“" sind demnach die Karten „1h“ und „1i“, die sich auf www.atlas-alltagssprache.de über die linke Navigationsleiste in der „Neunten Runde“ unter dem Stichwort Brötchen/Semmel finden. Alle Karten zu den Varianten lassen sich auch über das Register aufrufen.

5 „So vertreiben Sie wirkungsvoll Mücken und Schnaken“ (Stuttgarter Nachrichten Matthias Kemter, 04.06.2020) https://www.stuttgarter-nachrichten.de/inhalt.was-hilft-gegen-muecken-top-10hausmittel-mhsd.648ef052-e2d5-43cd-b605-caed69b3c322.html (abgerufen am 8.12.2020).

6 Duden-Wb.: Schnake: „1. (zu den Mücken gehörendes) Insekt mit schlankem Körper, langen, dünnen Beinen und Fühlern und schmalen Flügeln, das sich von Pflanzensäften ernährt” (abgerufen am 8.12.2020). 
2019, S. 763). Selbst bei Brötchen und Semmel ist zu beobachten, dass in Bäckereien beide Bezeichnungen für verschiedene Backwaren verwendet werden. Unterschiede zwischen regional verschiedenen Prototypen des Konzepts werden dabei zu distinktiven semantischen Merkmalen, weil auch die entsprechenden regionalspezifischen Varianten des Bezeichneten zu überall nebeneinander erhältlichen Spielarten des Produkts werden. So lernen SprecherInnen aus dem Brötchen-Gebiet in der heimischen Bäckerei, dass bestimmte Typen von Brötchen Semmel zu nennen sind - etwa Kaisersemmel als Bezeichnung einer aus Österreich übernommenen Gebäcksorte.

Wie der bekannt gewordene Fall Marmelade/Konfitüre zeigt, werden solche Differenzierungen durch die SprecherInnen bzw. VerbraucherInnen allerdings nicht immer akzeptiert: Die terminologische Regelung der EU mit Bedeutungsdifferenzierung nach den verarbeiteten Früchten (Zitrusfrüchte vs. andere) wurde von Österreich explizit abgelehnt, aber auch in Deutschland ist - entgegen den Etikettenbeschriftungen auf den Gläsern - unabhängig von der Fruchtsorte die allgemeine Bezeichnung Marmelade geblieben (vgl. AdA 12-1g). Das Scheitern der Bedeutungsdifferenzierung, von oben' kann in diesem Fall auch damit zu tun haben, dass die Variante Konfitüre außerhalb der Schweiz nirgends gebräuchlich war. Wie auch bei Dubletten wie den Pluralformen Wörter vs. Worte oder der Unterscheidung zwischen derselbe vs. der gleiche sichtbar wird, die zu Dauerbrennern der ,Zweifelsfälle“-Literatur geworden sind, sind der semantischen Ausdifferenzierung im allgemeinen Sprachgebrauch aber offensichtlich auch Grenzen der Ökonomie gesetzt.

\subsection{Standardsprache oder Alltagssprache?}

Wie im Fall Mücke/Schnake zu sehen war, sind die regionalen Markierungen in Wörterbüchern bei erhaltener Heteronymik nicht immer symmetrisch bei allen Varianten zu finden. Die unmarkierten Varianten werden dadurch als überregional standardsprachlich üblich dargestellt. Spielen bei der Vergabe solcher Markierungen wohl auch Entscheidungen der Kodifikator*innen eine Rolle ${ }^{7}$, so geht das Variantenwörterbuch einen anderen Weg und stellt die regionale und nationale Variation im standarddeutschen Wortschatz systematisch auf der Grundlage von Korpusanalysen dar; aufgrund ihres Vorkommens vor allem in Zeitungskorpora werden die aufgeführten Varianten im Prinzip als standardsprachlich eingestuft. Auch hier werden allerdings die Urteile von Sprachexpert*innen und die Markie-

7 Eine Erklärung zur Basis der Verbreitungsangaben im Duden-Wb. findet sich auf der Seite nicht. Das DWDS bietet seit kurzem ein nach den Arealeinteilungen von VWB und VG geordnetes Korpus, vgl. https://www.dwds.de/d/korpora/regional (abgerufen am 13.12.2020); allerdings scheint noch keine systematische Auswertung stattgefunden zu haben, die die regionalen Markierungen des digitalen DWDS präzisieren würde. 
rungen in Wörterbüchern einbezogen, um „Grenzfälle des Standards“ zu identifizieren (Indizien für diesen Status sind im Korpus dann z.B. eine Verwendung in Anführungszeichen oder eine Verwendung in konzeptionell mündlichen Textteilen) (vgl. VWB, 2016, S. XIIIf.). Solche Differenzierungen sind sicherlich der Tatsache angemessen, dass auch Laien-Sprachbenutzer*innen zwischen ,hochsprachlichen' und ,umgangssprachlichen' Ausdrücken unterscheiden. Die Gefahr eines Zirkels von Präskription - Schulunterricht - Sprecherurteilen - Deskription der Urteile - präskriptiver Rezeption der Deskription ist hier allerdings gegeben.

Für die Praxis macht schon das in Abschnitt 2.1 aufgeworfene Problem der Suchbegriffe im Online-Handel ${ }^{8}$ deutlich, dass auch in überregionalen Kontexten die Frage des konkreten Gebrauchs in verschiedenen Regionen - ungeachtet der Einstufung als Regionalismus, „Grenzfall des Standards“oder Standard - heute noch relevant ist. Dies gilt ebenso für den Unterricht von Deutsch als Fremdsprache, der den ersten Anlass für den Wortatlas der deutschen Umgangssprachen (im Folgenden kurz: WDU) ${ }^{9}$ gab. Der Atlas zur deutschen Alltagssprache (AdA) schließt sich dem Ansatz des WDU an: Es geht um den Gebrauch, der in den verschiedenen Regionen im Alltag üblich ist, unabhängig von der Frage nach dessen Standardnähe oder-ferne. Der Unterschied zwischen AdA und WDU liegt nur darin, dass die Daten für den AdA nicht mittels schriftlicher Befragung einzelner ausgewählter InformantInnen erhoben werden, sondern per Crowdsourcing über das Internet. Die seit 2003 durchgeführten zwölf Erhebungsrunden haben bisher Karten zu 598 vorwiegend lexikalischen, aber auch morphosyntaktischen, phonologischen, phraseologischen (vgl. Elspaß, 2020) und pragmatischen Variationsphänomenen erbracht. Viele dieser Phänomene sind aus der dialektalen Heteronymik ,ererbt', meistens nicht unverändert (vgl. Möller \& Elspaß, 2019, S. 760-763): Im Vergleich mit Dialektkarten zeigt sich die Zahl der Varianten in der Regel reduziert, insbesondere kleinräumig verbreitete sind durch solche ersetzt, die schon im Dialekt größere Verbreitungsareale haben, und diejenigen Varianten, die als ,standardsprachlich gelten, haben sich weiter ausgebreitet. Der Vergleich zwischen Karten des AdA und solchen des WDU bestätigt die anhaltende Tendenz zum Abbau von (insbesondere kleinräumigen) Varianten auch für die Entwicklung der Alltagssprache in den dazwischen liegenden ca. 40 Jahren (z.B. das Verschwinden von Potacken, Knollen, Bodenbirnen und Nudeln zugunsten von Kartoffeln, vgl. WDU I-75 ${ }^{10}$ vs. AdA 9-1e; weitere Beispiele in Elspaß, 2005, S. 7-10). Gleichzeitig zeigt sich jedoch auch hier weiterhin, dass großräumige Gegensätze häufig auch noch in der Alltagssprache sehr stabil sind.

8 Vgl. z.B. https://www.onlinemarketing.at/seo-lexikon/keyword-recherche/keywords-sammeln/ (abgerufen am 13.12.20).

9 Eichhoff $(1977,1979,1993,2000)$.

${ }^{10}$ Die römische Zahl bezieht sich auf den Band des WDU, die arabische Zahl auf die Kartennummer. 


\section{Neuere Variationsfälle}

\subsection{Nationale Varianten}

Die bisher genannten Beispiele arealer Variation im Deutschen gehen auf die dialektale Heteronymik zurück und damit auf die Herausbildung von arealen Unterschieden (dazu vgl. Lötscher, 2017) in einer Zeit erheblich beschränkteren überregionalen Austauschs. Mit der allgemeinen Verbreitung zunächst des Zugangs zu gedruckten Massenmedien durch die Schulpflicht, dann mit dem Aufkommen von überregionalen Tonmedien (Radio, Fernsehen) und schließlich mit den digitalen Medien hat sich die Situation für die Verbreitung sprachlicher Varianten deutlich geändert. Überregionale, passiv rezipierte Sprachvorbilder sind nicht nur allgemein zugänglich, sondern im Lauf von ca. 30 Jahren omnipräsent geworden. Schmidt und Herrgen (2011, S. 32) definieren „Makrosynchronisierung“ als die Annäherung des sprachlichen Wissens und Gebrauchs von nicht unbedingt persönlich miteinander kommunizierenden Sprechenden durch Ausrichtung an einer gemeinsamen Norm, sie beziehen sich damit in erster Linie auf die Standardnorm und deren Erwerb vor allem im schulischen Kontext. Eine ansatzweise ähnliche Wirkung entfaltet darüber hinaus auch die Ausrichtung an gemeinsamen Sprachvorbildern in den Medien. „Makrosynchronisierung“ hat damit eine viel größere Bedeutung gewonnen als in der Vergangenheit, wo Veränderungen sprachlicher (dialektaler) Konventionen im Wesentlichen über direkte Interaktionen („Mesosynchronisierung“ im Rahmen einer Gruppe S. 30) zustande kamen.

Die Erhaltung oder Entstehung von diatopischen Unterschieden zwischen sprachlichen Konventionen ist dementsprechend in neuerer Zeit grundsätzlich weniger begünstigt - mit Ausnahme vor allem der nationalen Ebene, wo eine unterschiedliche Variantenwahl in offiziellen Texten oder in nationalen Massenmedien unter modernen Bedingungen gerade besonders folgenreich ist. Diverse Studien zur Grenzdialektologie haben gezeigt, dass die Bezeichnungen für moderne Konzepte solche ,nationalen“ Muster bilden (vgl. z.B. Cornelissen, Schaars, \& Sodmann, 1993) und dass Staatsgrenzen sich auch darüber hinaus zu Bruchstellen in Dialektkontinua entwickelt haben (vgl. z.B. verschiedene Beiträge in Palliwoda, Sauer, \& Sauermilch, 2019).

Solche TendenzenzurAnnäherung sprachlicher Unterschiede an die Staatsgrenzen sind z.B. in der Entwicklung der Bezeichnungen für eine 'wollene Kopfbedeckung' deutlich zu erkennen: Kappe, ehemals überall im Südwesten Deutschlands üblich, ist heute in Deutschland weitestgehend von Mütze verdrängt und so beinahe schon zu einer rein Schweizer und (bislang) westösterreichischen und Südtiroler Variante geworden, in Österreich scheint jedoch Haube von Osten her Kappe zu ersetzen (vgl. AdA 8-3a gegenüber dem Deutschen Wortatlas ${ }^{11}, 12$, Kt. 7).

\footnotetext{
${ }^{11}$ Bearbeitet von Mitzka, Schmidt und Hildebrandt (1951-1980).
} 
Darüber hinaus können nationale Strukturen für die Entstehung spezifischer Varianten sorgen, zunächst einmal unmittelbar, durch Verwendung spezifischer Termini im Bereich der Justizund Verwaltung, des Schulwesens, der Verkehrsordnung usw. (Das VWB ist voller entsprechender Beispiele.) Wenn etwa der Deutsche Gewerkschaftsbund Informationen zum Thema Brückentag veröffentlicht, der Österreichische Gewerkschaftsbund dagegen zum Thema Fenstertag ${ }^{12}$, trägt dies sicherlich zum nationalen Erscheinungsbild der Verbreitungsareale der Varianten bei (AdA 11-5b; die oberösterreichischen InformantInnen geben allerdings geschlossen Zwickeltag an).

Die Rolle nationaler Institutionen und Strukturen hat auch nationale Verbreitungsbilder in Fällen von puristischem Fremdwortersatz bewirkt. So wurde z.B. im deutschen Kaiserreich die Ablösung zahlreicher Fremdwörter entscheidend durch den Einfluss des Allgemeinen Deutschen Sprachvereins auf staatliche Institutionen (Bahn, Post) oder zentral organisierte Vereine (Sport) gefördert (von Polenz, 1999, S. 267-274): Bahnsteig, Briefumschlag oder Elfmeter sind heute die einzig üblichen Varianten in Deutschland; in der deutschsprachigen Schweiz blieben dagegen Perron, Couvert und Penalty in Gebrauch. Eine derartige Verdeutschung ist auch Fahrrad: Zunächst waren die Fremdwörter Velociped $<$ frz. vélocipède und - besonders im Radsport-auch Bicycle in Gebrauch. Auf dem Velocipedisten-Congress 1884 wurde dann jedoch der Deutsche Radfahrerbund gegründet. Die AdA-Karte 'Fahrrad' (AdA 12-6c) zeigt dementsprechend einen klaren nationalen Gegensatz: In der Schweiz (sowie in Luxemburg und im Elsass) wird Velo verwendet, im übrigen deutschen Sprachgebiet Fahrrad oder Rad(l) (vgl. Abb. 1).

Nationale Muster treten auch bei der Verbreitung von Deonymen auf. So hat sich speziell in der Schweiz Bostitch für das 'Gerät zum Anbringen von Heftklammern' etabliert (AdA 8-3g, vgl. Abb. 2), nach dem Namen der USamerikanischen Bostitch Sales Company (aus Boston Wire Stitcher). Ebenso beschränkt sich die Bezeichnung der 'Gläser zur luftdichten Konservierung von Lebensmitteln' als Rexglas - nach dem Hersteller Gustav Rex - auf Österreich (ohne Tirol und Vorarlberg) (AdA 7-5a). Ein Grund hierfür könnten national und regional spezifische Absatzmärkte sein. Eine solche nationale Spezifik fehlt allerdings bei der ebenfalls deonymischen Bezeichnung Einweckglas - nach dem Unternehmer Johann Carl Weck -, die in einem langen Streifen von der Ostsee bis nach Südtirol dominiert.

12 https://www.dgb.de/++co++97ac89be-fb1e-11e9-8da0-52540088cada vs. https://www.oegb.at/ cms/S06/S06_0.a/1342622634730/home/so-kann-man-die-feiertage-2020-richtig-nuetzen (abgerufen am 8.12.2020). 


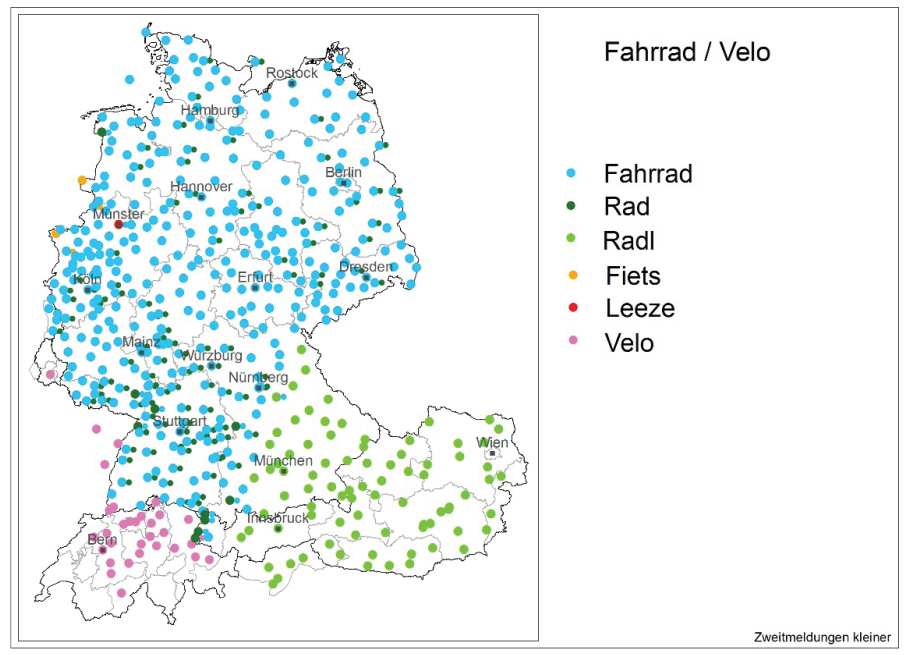

Abbildung 1: Bezeichnungen für ein ,nicht motorisiertes Zweirad‘ (AdA 12-6c)

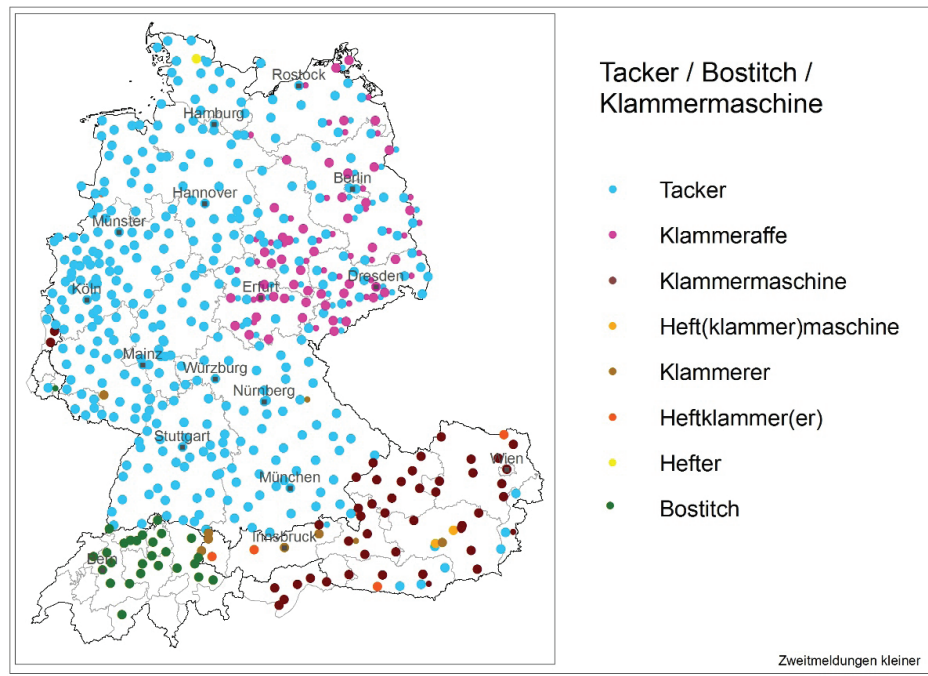

Abbildung 2: Bezeichnungen für ein ‘Gerät zum Anbringen von Heftklammern’ (AdA 8-3g)

Nationale Verbreitungsbilder bei neueren Konzepten kommen aber auch ohne konkrete Beteiligung nationaler Instanzen oder Strukturen zustande, wie Autodrom (Österreich) und Putschauto (Schweiz) gegenüber Auto-Scooter zeigen (AdA 2-f12). Und auch bei neueren Konzepten ist noch zu beobachten, wie sich wie im Fall 'Mütze' - Varianten zunehmend zu nationalen Varianten entwickeln: In der WDU-Karte 'Aufzug' (IV-4) ist Lift zwar in Österreich und der Schweiz klar 
dominant, aber zumindest als Zweitbeleg auch in Deutschland überall verstreut und in Bayern sogar häufig, umgekehrt ist Aufzug auch in Österreich nicht selten. In der AdA-Karte (12-6g) dagegen kommt Lift in Deutschland praktisch nicht mehr vor, und Aufzug in Österreich nur noch ganz im Osten ein paarmal.

Angesichts der schon angeführten Faktoren ist es naheliegend, dass viele neuere lexikalische Variationsfälle eine Konzeption des Deutschen als plurizentrische Sprache stützen und sogar einige ältere sich in Richtung nationaler Verbreitungsmuster entwickelt haben (und dies weiter tun), wie die Beispiele zeigen. Dagegen einzuwenden ist allerdings zunächst einmal die große Zahl von mehr oder weniger stabilen arealen Verteilungen, bei denen die deutsche Südhälfte nicht mit der Nordhälfte, sondern mit Österreich und teilweise der Schweiz eine Einheit bildet (vgl.auch unten). Darüber hinaus ist auffällig, dass in den meisten der angeführten Beispielkarten über die nationalen Gegensätze hinaus auch kleinräumige bzw. regional verbreitete Varianten auftreten. Es kommt auch bei diesen neueren Konzepten offensichtlich weiterhin zu Innovationen und arealer Durchsetzung von Varianten unterhalb der nationalen Ebene (zur Diskussion um Plurizentrizität und Pluriarealität des Deutschen vgl. etwa Sutter, 2017, S. 31-38).

\subsection{Neue regionale Varianten}

Natürlich können die genannten steuernden Faktoren auch unterhalb der nationalen Ebene wirksam werden. Wo z.B. das Schulwesen auf Bundesländeroder kantonaler Ebene geregelt ist, können auch hieraus entsprechende Variationsmuster entstehen, die nur innerhalb dieser politischen Räume gelten, wie an den Bezeichnungen für eine 'angekündigte Schulprüfung' ersichtlich ist: Eine solche heißt (nur) in Bayern Schulaufgabe, in westlichen Kantonen der Deutschschweiz Probe, in den östlichen Kantonen der Deutschschweiz Prüfung (bzw. Prüfig, AdA 5-11a-b).

Es gibt darüber hinaus aber offensichtlich weiterhin eine regionale Dynamik in der Herausbildung und Verbreitung von Varianten. So zeigt die Karte 'Fahrrad' nicht nur den erwähnten Gegensatz zwischen Velo und der Verdeutschung Fahrrad, sondern auch ein klar abgegrenztes homogenes Areal im Südosten, und zwar ziemlich genau im Gebiet der bairisch-österreichischen Dialekte (Altbayern, Österreich ohne Vorarlberg, Südtirol): Dort ist nicht die Form (Fahr-)Rad, sondern das Diminutiv Radl üblich. In der Karte 'Leitkegel' (AdA 11-4a) hebt sich nicht nur Töggeli als Schweizer Variante von Verkehrshütchen im übrigen Gebiet ab; darüber hinaus wurde in einem klar umrissenen (vor allem alemannischen) Gebiet in Südwestdeutschland einhellig Hütle statt Verkehrshütchen angegeben. Die jeweiligen Areale dieser spezifischen diminuierten Formen verweisen natürlich auf die Verteilung der Diminutivformen (vgl. auch 'Mädchen', AdA 8-5e), aber die Verwendung der diminuierten Formen ohne die Bestimmungswörter Verkehrs- bzw. Fahr- entspricht offenbar Konventionen, die sich in den beiden 
Fällen unterschiedlich gebildet haben. Deutlich ist allerdings in beiden Fällen gleichzeitig auch, dass weitere Varianten - die undiminuierte Kurzform Rad und die Variante Pylon 'Leitkegel' - zwar regionale Schwerpunkte erkennen lassen, aber keine wirklich abgrenzbaren Areale bilden und durchgehend nur neben der insgesamt dominierenden Variante erscheinen. Festere regionale Konventionen sind hier nicht (nicht mehr oder noch nicht?) entstanden.

Eine anhaltende Koexistenz von Varianten zeigt sich auch bei 'Aufzug' (AdA 12-6g) mit dem Nebeneinander von Fahrstuhl und Aufzug in der Pfalz und in Westfalen, das schon die WDU-Karte (4-4) in diesen Gebieten abbildet. Im Rest von Deutschland stehen sich jedoch klar abgegrenzt Fahrstuhl in Nord- und Ostdeutschland und Aufzug im übrigen Gebiet gegenüber.

Auf eine rezente Weiterentwicklung in Richtung einer regionalen Konsolidierung deutet der Vergleich der AdA-Karte Bürgersteig/Gehsteig (AdA 11-4g) mit der entsprechenden Karte des WDU (WDU 1-30) hin. Auch hier handelt es sich um die Koexistenz eines Lehnworts (Trottoir) und mehrerer deutscher Ersatzwörter. Und auch hier ist in der Schweiz im WDU wie im AdA allein das Lehnwort verzeichnet. Außerhalb der Schweiz ist in der Karte I-30 des WDU das Bild jedoch noch relativ gemischt. Der Vergleich von WDU- und AdA-Karte zeigt insgesamt, dass die in den 1970er Jahren noch relativ unscharf begrenzten Verbreitungsgebiete der Varianten bis heute an Kontur(en) gewonnen haben (Gehsteig in Bayern, Österreich und Südtirol, Gehweg in Baden-Württemberg, Fußweg in Sachsen und Sachsen-Anhalt, Bürgersteig v.a. im Norden und Westen Deutschlands). Auf eine zunehmende überregionale Vereinheitlichung deutet der Vergleich also nicht hin, im Gegenteil: Die Verbreitung stabiler Varianten scheint sich häufig (bundes-) landesweit bzw. bundeslandweit zu konsolidieren.

Im Fall der Bezeichnungen für einen 'Rettungswagen' (AdA 3-14g) zeigen sich wieder nationale Unterschiede (Rettung nur in Österreich und Südtirol, Ambulanz nur in der Schweiz und Ostbelgien/Luxemburg), aber umrisshaft auch ein spezifisches Areal in Bayern und Teilen Schwabens, das auf horizontale Ausbreitung hinweist: Die Variante Sanka (< Sanitätskraftwagen) aus dem (überregionalen) militärischen Gebrauch hat sich in dieser Region - und nur dort - auch breit in der Alltagssprache etabliert.

Eine (zumindest umrisshafte) Arealbildung bei rezenten informellen bzw. scherzhaften Bezeichnungen für relativ neue Konzepte findet sich schließlich auch bei der Bezeichnung Klammeraffe für die Klammermaschine (AdA 8-3g, vgl. Abb. 2) und bei Tischtenniskelle für 'Tischtennisschläger' (AdA 8-3d): Beide kommen in den Karten nur in Ostdeutschland vor, sind dort jedoch flächendeckend präsent. Ebenso scheint eine regional beschränkte Ausbreitung eines Neologismus beim lautmalerischen Bim 'Straßenbahn' vorzuliegen: In der Karte des WDU (I-47) erscheint hierzu eine einzige Sondermeldung in der Steiermark, in der Karte des AdA (12-6f, vgl. Abb. 3) von 2020 ist Bim schon im 
ganzen Ostteil von Österreich verbreitet. Und als regionale Bezeichnungen für den 'Autoscooter' sind Komposita entstanden, die auf unterschiedliche dialektale oder regiolektale Verben mit der Bedeutung 'aufprallen' zurückgreifen (wie ja auch das schweizerische Putschauto): Knuppauto (Eifel/Mosel) und Boxauto (Südwestdeutschland).

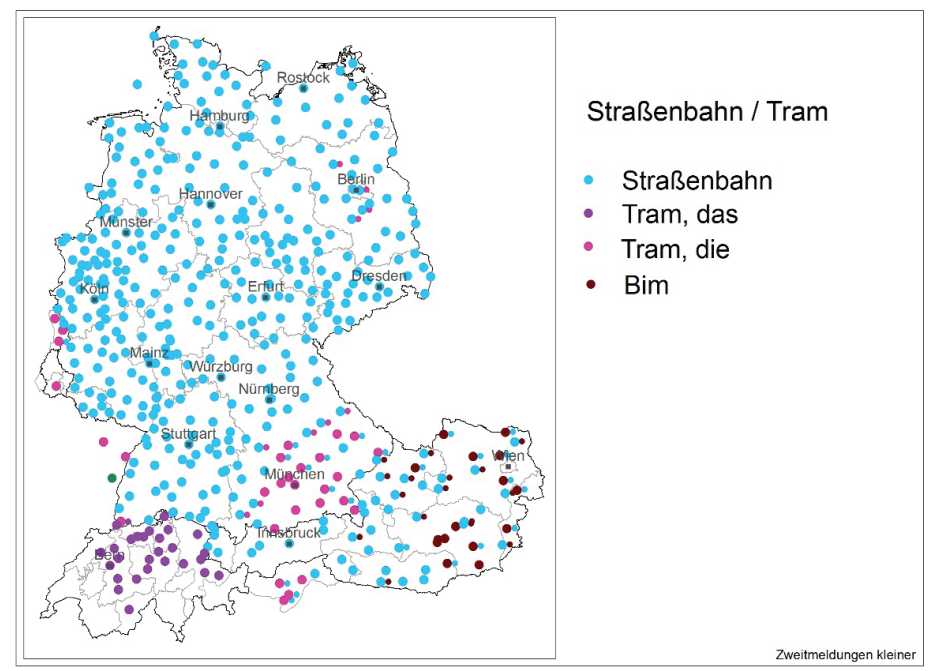

Abbildung 3: Bezeichnungen für die 'Straßenbahn' (AdA 12-6f)

\section{Heute dominierende Arealstrukturen}

Die angeführten Karten zur Variation bei relativ jungen Konzepten lassen deutlich wiederkehrende Muster erkennen: Bei einer großen Zahl von ihnen zeigt sich eine volle oder weitgehende Übereinstimmung von Geltungsarealen von Varianten mit Staatsgebieten, was zunächst einmal die Überlegungen zur Bedeutung nationaler Diffusionswege im 20. Jahrhundert bestätigt. Darüber hinaus aber ist in all diesen Karten auch (zumindest im Ansatz) eine Arealbildung unterhalb der nationalen Ebene festzustellen, mit spezifischen Varianten v.a. im Südwesten (beispielsweise Hütle), Südosten (Schulaufgabe) und Osten Deutschlands (Klammeraffe) - oder auch in Oberösterreich (Zwickeltag). Hinzu kommen grenzüberschreitende Gemeinsamkeiten entgegen den national dominierenden Varianten, z.B. in Tirol und Vorarlberg, wo die regionalen alemannisch geprägten - Varianten häufig mit Varianten in der benachbarten Schweiz und in Liechtenstein übereinstimmen.

Auf vielen AdA-Karten ist dagegen zu sehen, dass bei älteren Phänomenen eher großräumige Nord-Süd-Unterschiede im Vordergrund stehen, wo Deutschland in der Mitte geteilt erscheint. In summarischen Auswertungen von verschiedenen Teilen des AdA-Korpus (wie schon des WDU-Korpus, vgl. Möller, 2003) mit verschiedenen 
dialektometrischen Verfahren ${ }^{13}$ haben sich jedenfalls jedes Mal genau diese Kontraste als quantitativ dominierend erwiesen: Prägend für die alltagssprachliche Variation heute sind danach vor allem die Staatsgrenzen einerseits und der großräumige NordSüd-Gegensatz im Bereich der „Mainlinie“ andererseits. In der Faktorenanalyse von Pickl und Pröll (2019, S. 875) zeigt sich die Staatsgrenze zwischen Österreich und Deutschland (bzw. Bayern) dabei weniger prominent als die zwischen der Schweiz und den Nachbarländern. In allen Untersuchungen erweist sich allerdings die großräumige Nord-Süd-Teilung gegenüber den nationalen Gegensätzen im oberdeutschen Raum als noch wichtiger. Die Mainlinie, welche die Alltagssprache in eine eher nördliche und eine eher südliche sprachliche ,Hemisphäre teilt, hat interessanterweise eine Entsprechung im sprachlich-kulturellen Bewusstsein der Bevölkerung: Durrell (1989) verweist darauf, dass diese Linie mit der Grenze des Norddeutschen Bunds im 19. Jahrhundert übereinstimmt und damit im damaligen politischen Diskurs die Südgrenze des preußischen Einflussbereichs markierte; sie hat sich damit als wichtige Grenzlinie in der ,Mental Map“ der Bevölkerung eingeprägt - und wirkt offenbar bis in die Gegenwart hinein.

\section{References}

Ammon, U., Bickel, H., Lenz, A. N., Fink, J., Gellan, A., Hofer, L. ... Suter, S. (2016). Variantenwörterbuch des Deutschen. Die Standardsprache in Österreich, der Schweiz, Deutschland, Liechtenstein, Luxemburg, Ostbelgien und Südtirol sowie Rumänien, Namibia und Mennonitensiedlungen (2nd. ed., text rev.). Berlin, Boston: De Gruyter. DOI: 10.1515/9783110245448.

Cornelissen, G., Schaars, A., \& Sodmann, T. (Eds.) (1993). Dialekt à la carte. Dialektatlas Westmünsterland - Achterhoek - Liemers - Niederrhein. Doetinchem, Vreden, Köln: Rheinland-Verlag.

Duden Wörterbuch. Retrieved December 12, 2020, from https://www.duden.de/.

Durrell, M. (1989). Die „Mainlinie“ als sprachliche Grenze. In W. Putschke, W. Veith, \& P. Wiesinger (Eds.), Dialektgeographie und Dialektologie (pp. 89-109). Marburg: N. G. Elwert.

Eichhoff, J. (1977). Wortatlas der deutschen Umgangssprachen: Vol. 1. Bern: Francke.

Eichhoff, J. (1978). Wortatlas der deutschen Umgangssprachen: Vol. 2. Bern: Francke.

Eichhoff, J. (1993). Wortatlas der deutschen Umgangssprachen: Vol. 3. München: Saur.

Eichhoff, J. (2000). Wortatlas der deutschen Umgangssprachen: Vol. 4. Bern, München: Saur.

Elspaß, S., \& Möller, R. (2003-). Atlas zur deutschen Alltagssprache (AdA). Retrieved December 12, 2020, from www.atlas-alltagssprache.de.

Elspaß, S. (2005). Zum Wandel im Gebrauch regionalsprachlicher Lexik: Ergebnisse einer Neuerhebung. Zeitschrift für Dialektologie und Linguistik, 72(1), 1-51.

Elspaß, S. (2020). Areal Variation and Change in the Phraseology of Contemporary German. In E. Piirainen, N. Filatkina, S. Stumpf, \& C. Pfeiffer (Eds.), Formulaic Language and New Data: Theoretical and Methodological Implications (pp. 43-77). Berlin, Boston: De Gruyter. DOI: 10.1515/9783110669824-003.

Hartweg, F., \& Wegera, K. P. (1989). Frühneuhochdeutsch: Eine Einführung in die Sprache des Spätmittelalters und der frühen Neuzeit. Tübingen: Niemeyer.

13 Clusteranalyse von 224 AdA-Karten in Lang (2008, vgl. auch Möller \& Elspaß, 2019, S. 778) sowie Faktorenanalyse von 245 anderen AdA-Karten in Pickl und Pröll (2019, S. 875). 
Kleiner, S. (2011-2021.). Atlas zur Aussprache des deutschen Gebrauchsstandards (AADG). With the collaboration of R. Knöbl. Retrieved December 12, 2020, from http://prowiki.idsmannheim.de/bin/view/AADG/.

Lang, B. (2008). Zur dialektometrischen Analyse des Atlas zur deutschen Alltagssprache (Admission thesis, Universität Augsburg, Germany). Retrieved December 12, 2020, from www.atlasalltagssprache.de/literatur.

Lötscher, A. (2017). Areale Diversität und Sprachwandel im Dialektwortschatz. Untersuchungen anhand des Sprachatlas der deutschen Schweiz. Stuttgart: Steiner.

Mitzka, V., Schmidt, L. E., \& Hildebrandt, R. (1951-1980). Deutscher Wortatlas: Vol. 22. Gießen: Schmitz.

Möller, R. (2003). Zur diatopischen Gliederung des alltagssprachlichen Wortgebrauchs: Eine dialektometrische Auswertung von Jürgen Eichhoff: „Wortatlas der deutschen Umgangssprachen“ (Vols. 1-4; 1977, 1978, 1993, 2000). Zeitschrift für Dialektologie und Linguistik, 70(3), 259-297.

Möller, R., \& Elspaß, S. (2019). Die rezente Dynamik im arealsprachlichen Lexikon. In J. Herrgen, \& J. E. Schmidt (Eds.), Sprache und Raum. Ein internationales Handbuch der Sprachvariation: Vol. 4. Deutsch (pp. 756-781). Berlin, Boston: De Gruyter. DOI: 10.1515/9783110261295-025.

Palliwoda, N., Sauer, V., \& Sauermilch, S. (Eds.) (2019). Politische Grenzen-Sprachliche Grenzen? Dialektgeographische und wahrnehmungsdialektologische Perspektiven im deutschsprachigen Raum. Berlin, Boston: De Gruyter. DOI: 10.1515/9783110571110.

Pickl, S., \& Pröll, S. (2019). Ergebnisse geostatistischer Analysen areal-sprachlicher Variation im Deutschen. In J. Herrgen, \& J. E. Schmidt (Eds.), Sprache und Raum. Ein internationales Handbuch der Sprachvariation: Vol. 4. Deutsch (pp. 861-879). Berlin, Boston: De Gruyter. DOI: 10.1515/9783110261295-030.

Polenz, P. von (1999). Deutsche Sprachgeschichte vom Spätmittelalter bis zur Gegenwart: Vol. 3. 19. und 20. Jahrhundert. Berlin, New York: De Gruyter. DOI: 10.1515/9783110805918.

Schmidt, J. E., \& Herrgen, J. (2011). Sprachdynamik: Eine Einführung in die moderne Regionalsprachenforschung. Berlin: Erich Schmidt Verlag.

Sutter, P. (2017). Diatopische Variation im Wörterbuch: Theorie und Praxis. Berlin, Boston: De Gruyter. DOI: 10.1515/9783110482263.

Variantengrammatik des Standarddeutschen (2018). An open access online reference work compiled by a team under the leadership of C. Dürscheid, S. Elspaß \& A. Ziegler. Retrieved December 12, 2020, from http://mediawiki.ids-mannheim.de/VarGra. 